\title{
Prospective study on intracranial pressure waveform morphology and its relationship with the development of obesity in rats
}

\author{
Diego Adorna Marine ( $\nabla$ diegomarine@gmail.com ) \\ Federal University of São Carlos \\ João Manoel Alves \\ Federal University of São Carlos
}

Marcela Sene-Fiorese

Federal University of São Carlos

Cristina Arrais

Federal University of São Carlos

Stela Marcia Mattielo

Federal University of São Carlos

Jeferson Ancelmo Teixera

Federal University of São Carlos

Vanessa Oliveira Furino

Federal University of São Carlos

Carla Nascimento Rodrigues dos Santos

Federal University of São Carlos

Ricardo Carneiro Borra

Federal University of São Carlos

Ana Claudia Garcia Oliveira Duarte

Federal University of São Carlos

\section{Research Article}

Keywords: Intracranial Pressure Waveform, Arterial Hypertension, Heart Rate, Obesity and Body Composition

Posted Date: March 24th, 2021

DOl: https://doi.org/10.21203/rs.3.rs-353495/v1

License: (c) (1) This work is licensed under a Creative Commons Attribution 4.0 International License. Read Full License 


\section{Abstract}

Obesity accumulates fat in the body may cause hypertension and change intracranial pressure waveform morphology (ICP). However, not clear how and when it occurs. Thus, our objective was to evaluate the ICP waveform morphology and P2/P1 ratio during the development of obesity. Sixteen 45-day-old male Wistar rats, after 60 days of adaptation were divided into non-obese $(N O B, n=7)$ and obese $(O B, n=9)$. The OB group received a high-fat diet and the NOB group received a standard diet for 24 weeks. ICP, body composition, blood pressure (BP), and heart rate(HR) were assessed every 4 weeks. Two-Way ANOVA was used for the area under the curve with Bonferroni's test, or Student's t-test for BP, and HR. Body mass(BM) and body fat(BF) and fat-free mass(FFM) were higher in $\mathrm{OB}$ rats. There was no difference in $\mathrm{BP}$, but there was a difference in HR, greater in OB. The ICP decreased by NOB. These results suggest that the increase in BM and BF caused obesity, and BM can explain the increase in FFM. Although we did not find pathological states of ICP, NOB demonstrated better brain compliance, and the upward trend in the P2 waveform in $\mathrm{OB}$ may indicate impairment to the ICP of $\mathrm{OB}$ rats.

\section{Introduction}

Obesity is a multifactorial disease, resulting from the low energy expenditure associated with a diet high in lipids and carbohydrates. Its progression has been related to the development of several pathologies, such as arterial hypertension (AH), dyslipidemia, insulin resistance, type II diabetes mellitus ${ }^{1}$, and increased intracranial pressure (ICP) ${ }^{2-4}$.

ICP is the pressure exerted inside the cranial box derived from the interaction of three main components: brain, cerebrospinal fluid (CSF), and cerebral blood volume (CBV). Changes that result in volume imbalance can cause intracranial hypertension $(\mathrm{ICH})^{5,6}$. This increase may lead to a stroke, spontaneous intracranial hemorrhage, and even death ${ }^{7}$. Epidemiological studies have shown that there is a strong relationship between obesity and ICP, and one of the possible mechanisms for ICP alteration is due to chronic inflammation caused by obesity ${ }^{8-10}$. In the same way, studies have shown that weight loss improves the ICP of obese patients ${ }^{9,10}$. Thus, monitoring of ICP in overweight and obese individuals becomes essential in clinical practice ${ }^{11}$.

Part of the difficulty in analyzing ICP in humans lies within the method of analysis. Currently, the most reliable way to assess ICP is through an invasive high-cost procedure performed exclusively in hospitals. Therefore, alternative non-invasive methods facilitate ICP assessment without the need for a hospital environment, increasing the possibility of clinical evaluation ${ }^{12,13}$. Monro-Kelli's theory, demonstrated in 1783 , says that an increase in some volume inside the skull must result in the decrease of another. As the cerebral parenchyma usually has a constant volume, which can change due to traumatic brain injury for example, or another form of swelling, the volumes that change most frequently are blood and CSF, and blood increase would result in the decrease of $\mathrm{CSF}^{14,15}$. However, it has been demonstrated that there is cerebral compliance allowing blood to accumulate without decreasing $\operatorname{CSF}^{16}$; and the skull has small 
movements, allowing for ICP assessment using sensors sensitive to minimal dislocations ${ }^{12}$. Thus, the Braincare equipment and non-invasive sensor were used for validation in comparison to the invasive method, obtaining a strong correlation ${ }^{12,14}$.

The ICP waveform morphology can help with the diagnostic changed ICP in clinical analysis. The ICP waveform morphology has three pressure wave peaks detected, which correspond to P1, P2, and P3, and the physiological pattern must be $\mathrm{P} 1>\mathrm{P} 2$ > P3 in normal physiological states. When P1 is reduced, it shows loss of cerebral perfusion and the increase in $\mathrm{P} 2$ shows loss of brain compliance ${ }^{5,17-19}$. Four waveform shapes were previously identified, normal (class I), potentially pathological (class II), likely pathological (class III), and pathological (class IV), intensifying with the increase of P2 until the formation of a single peak, therefore being of great importance for the clinical evaluation of the patient ${ }^{2-6}$.

Thus, we hypothesize that the development of obesity can cause an increase in the P2 / P1 ratio in obese rats $(\mathrm{OB})$ in relation to non-obese rats $(\mathrm{NOB})$. The aim of this prospective study was to describe, for the first time, the behavior of the ICP waveform morphology non-invasively (ICPn) during the development of obesity in Wistar rats.

\section{Results}

\section{Body Composition}

The high-fat diet promoted an increase in body mass and the percentage of body fat. Figure $3 \mathrm{~A}$ shows the behavior of body mass over the 24 weeks of treatment, in which the obese rats $(\mathrm{OB})$ that started from the same condition but obtained a much greater body mass than the non-obese rats (NOB) and obesity was classified by BM at week 12. Two-way ANOVA demonstrated an interaction between the covariable time and groups $(F=10.42 ; p=0.0029)$; because non-obese animals also gain weight over time, meanwhile the diet applied to OB shows a greater increase in body mass over NOB, which can be observed with a significant increase from week $12(p<0.05)$ to week $24(p<0.001)$ shown in Fig. 3B.

Regarding BF, two-way ANOVA demonstrated no interaction between groups and the covariable time ( $\mathrm{F}=$ 3.394; $p=0.0747)$. However, $O B$ rats showed a large increase $(p<0.001)$ during the treatment period in relation to NOB, in which it remained constant. The difference grows even more from week 12 to week 24 observed through the increase of significance, as well as through body mass, and shown in Fig. $4 \mathrm{~B}$ and the highest fat accumulation in $O B$ is shown in Fig. $4 A$.

Still observing the body composition, there was also a significant increase of FFM in OB in relation to NOB, there being an interaction between groups and the covariable time $(F=5.576 ; p=0.0245)$. The development of FFM during the period can be seen in Fig. 5A. Although body mass start in the same initial condition, BF and FFM were different between groups in week 0 , as can be seen in Table 1. However, there was a significant increase between the groups between weeks 12 and 24, demonstrated by the statistic $(p<0.001)$ in Fig. 5B. 


\section{Intracranial Pressure}

Regarding P2 / P1 ratio of the ICP waveform, there was no interaction between groups and time ( $\mathrm{F}=$ 1,299; $p=0.2629$ ). However, NOB rats showed a below P2 / P1 ratio (Fig. 6B), during the treatment period compared to OB $(p=0.0001)$ between 12-24 weeks. The baseline value found at the beginning of the experiment, in the $\mathrm{OB}$ group, was maintained until the end of the experiment, meanwhile, as can be seen in Fig. 6A, the NOB group had a small decrease in week eight, and remaining with the lower values than baseline values in the sequence, and remaining below the OB group all the time of the experiment, shown in Table 1. Despite this difference, no statistical changes were found over time. Meanwhile, at week 12, there was a small increase in the P2 / P1 ratio in the OB group, seen in Fig. 7D. The Fig. 7B and 7D show the average pulse curve of the fifth minute in the NOB and OB groups, respectively, at week 24 . Some animals of $\mathrm{OB}$ group showed a ratio of 0.75 and remained with a fluctuation between 0.7 and 0.75 , which until the end of the experiment. However, this P2 /P1 ratio is presented as class I, normal. As can be seen in Table 1, by means of the standard deviation, the OB group showed greater data variability than the NOB group.

\section{Blood Pressure and Heart Rate}

Diastolic blood pressure (DBP), systolic blood pressure (SBP), and mean arterial pressure (MAP), obtained from week 12 on when obesity was established, did not show a statistical difference between groups as can be seen in Figs. 8A, 8B, and 8C, respectively. However, the HR of OB animals was higher than NOB in the same period $(p<0.05)$, as shown in Fig. 8D below.

\section{Discussion}

As previously demonstrated, $\mathrm{OB}$ rats fed a high-fat diet had a higher $\mathrm{BM}$ and $\mathrm{BF}$ than $\mathrm{NOB}^{22-26}$ rats; similar to the obesity phenotype in humans. Considering literature does not present a defined BMI for rats, and most studies present the issue of BF always related to the respective control groups, our body composition results consolidate our perspective of having a standard of rat body composition. Therefore, we used the same standard of reference for humans $\geq 20 \%$ overweight and $\geq 25 \%$ obese for men ${ }^{27}$, being an important reference value for future work with rats. At the end of the experiment, an average of very close to $25 \%$ of adult males can be observed, however, our data variability explains a little lower average, and the BM classifies them as obese ${ }^{27,28}$, and some animals that had presented BF above $25 \%$, being classified as obese through the BF. Thus, our results are in line with those in the literature, but we would like to observe these alterations over time in more detail, and a gold standard assessment such as the one carried out in the present study, brings this depth.

At week 12 , we identified BF close to $20 \%$ in OB rats classified as overweight, but the BM was similar to Ambrosi (2011) ${ }^{27}$ and Leopoldo (2016) ${ }^{28}$ at 15 weeks of obesity induction classified as obese, however, the diet used in the present study had a higher caloric index. Therefore, we believe that week 12 may be the ideal point for inducing obesity. It is important to highlight that the present study presented, at week $24, \mathrm{BM}$ of $\mathrm{OB}$ rats greater than that stipulated in literature 27,28 . 
With regard to FFM, we observed that the greatest increase in the OB group was due to the increase in muscle weight, as we collected the gastrocnemius, soleus, and EDL muscles, both right and left, and the sum of their weight was greater in $\mathrm{OB}$ at the end of the experiment, as well as the bone mineral content in the $\mathrm{OB}$ group was higher (shown in Figs. $1 \mathrm{a}$ and $1 \mathrm{~b}$, respectively, in supplementary information). These data corroborate with the literature carried out with humans ${ }^{29}$ and rats ${ }^{30}$ since obese people have greater muscle and bone overload. It is worth mentioning that, at the end of the experiment, these animals were middle-aged, and possibly with no signs of sarcopenic obesity appearing, which can affect both the loss of muscle and bone mass ${ }^{31}$.

Regarding ICP (P2 / P1) ratio of ICP waveform morphology, the difference found between the OB and NOB groups due to the decrease in NOB group is different from Uldall et al. (2017) ${ }^{32}$ who demonstrated an increase in aquaporin 1 (AQP1), causing an increase in CSF and consequently an increase in ICP. Meanwhile, in their study, they observed values of $7.70 \pm 0.42 \mathrm{mmHg}$ for OB and $5.86 \pm 0.15 \mathrm{mmHg}$ in NOB, which despite showing this difference, the values are normal when compared to humans ${ }^{5}$, similar in the present study that demonstrated ICP shape class I.

Despite the difference between OB and NOB in this study, no animal had a P2 / P1 > 1 ratio being considered potentially pathological or other class, through reference classification, therefore classified normal ${ }^{20}$. We cannot state the gross ICP value $(\mathrm{mmHg})$ of these animals due to the methodological limitation. There are several causes for the development of intracranial hypertension $(\mathrm{ICH})^{9}$, it being an idiopathic disease. However, studies have shown that overweight and obesity can lead to $\mathrm{ICH}^{8-10,33}$, even as the present study demonstrates that obesity can change the ICP waveform morphology (not confirmed by the present study) and that it can be an indicator of the change in ICP values, increasing the possibilities of diseases related both to obesity and to those resulting from increased ICP, as like stroke, blindness, among others.

In addition, when there is an increase in volume inside the cranial box and a decrease in brain elastance and compliance, ICP should increase ${ }^{6,16}$, and a small increase in volume to lead to a large increase in ICP. Thus, we infer the lower the P2 / P1 ratio in the NOB group, can be for the better the brain elastance and compliance than $\mathrm{OB}$ group, despite animals being sedated during ICP analyses. In the literature, magnetic resonance images demonstrate increased CSF in subdural spaces ${ }^{16}$ showing brain compliance, with $\mathrm{P} 1$ being related to the arterial influx, increasing CBV. However, the subsequent increase in ICP is linked to the CSF outflow, increasing arterial and venous flow interacting, changing the component cranial volume participation. Therefore, good compliance enables accumulation of another cranial volume without higher outflow of CSF, making it difficult to analyze and understand the hemodynamic of this system ${ }^{6}$ and hampering volume self-regulation. Meanwhile, cerebral compliance was not measured in the present study as well as the mean ICP value in $\mathrm{mmHg}$, and also the value peaks that comprise it (P1; P2; P3). Unfortunately, the system used in this study, as well as other non-invasive ones, does not allow us to know these values and we also did not evaluate the hemodynamic and/or elastance and compliance brain. 
Concerning BP, there was no statistical difference between OB and NOB, similar as Shiou et $\mathrm{al}^{34}(2018)$ found, and the absolute BP values $(\mathrm{mmHg}$ ) of the present study were similar to those found by Shiou et $\mathrm{al}^{34}$ (2018). However, their analysis was invasive, with animals sedated by pentobarbital, and in the present study, BP was performed on the same animals for 24 weeks, which could not be done invasively. Meanwhile, the volume pressure-recording (VPR) sensor used in the present study, has a moderate to high correlation with telemetry and direct blood pressure measurement ${ }^{35}$. Furthermore, the great variability of our data can also explain this lack of difference in BP. Uldal et al ${ }^{32}$ (2017) also demonstrated that there is no difference in $\mathrm{BP}$ between $\mathrm{OB}$ and $\mathrm{NOB}$ rats, and indicating that the increase in ICP is not related to the increase in BP and HR, as PA and HR did not change in their studies. However, in the present study, both groups were hypertensive and a difference was found between the HR groups. We found a significant difference in HR between $\mathrm{OB}$ and NOB rats, being greater in OB, different from Shiou et al ${ }^{34}$ (2018) and Uldal et al $\left.\right|^{32}$ (2017). These data corroborate those of Spereta et $\mathrm{al}^{36}$ (2018) that demonstrated to increase in HR with obese rats, with similar values to the present study. We believe that this difference may be related to increased cardiac work and respiratory rate (not assessed in this study) due to excessive weight. Therefore, may have a link between HR and ICP, despite being sedated during ICP assessment, and this relationship needs to be further studied.

\section{Conclusion}

We concluded that 12 weeks are necessary to start the development of obesity in rats, and from then on the differences in body composition become more evident, being confirmed in 24 weeks of treatment for BF values, through values similar to those presented in human beings. Moreover, we used the same reference values for humans and literature (for rats) in the present study. The fact that we evaluated this variable with DXA allowed us to monitor the fattening process in body fat percentage, in the same animal.

We have not confirmed our hypothesis about the influence of obesity on the ICP waveform morphology and an increase in the P2 / P1 ratio in OB group. Therefore, the difference developed over time might as well be due to increased compliance, and a decrease P2 / P1 ratio in NOB group. Meanwhile, the ICP waveform morphology in $\mathrm{OB}$ tends to be directed towards the potentially pathological state found in literature as a reference, because there were fluctuations over time, with P2 a small higher in OB, but normal class classified yet. Body composition can be an important risk factor for ICP changes and not time. BP does not differ between groups and time in this study, or does it influence ICP. Likewise, our results suggest that obesity causes an increase in HR compared to the control group.

The major limitation of our study is the absolute value of ICP not being performed by the device used, once it is also important to know the values of each peak. Another limitation was no cluster analysis was performed, which could demonstrate subgroups, due to different morphological characteristics of the groups, being important for reference standards for rat body composition. The sample size of the present study is considered small for this cluster analysis, being another limitation of the present study. In 
summary, this work is the first that attempts to elucidate the behavior of ICP waveform morphology noninvasively about the development of obesity, with a new proposal for the analysis of ICP, and also trying to help in elucidating the relationship between obesity, BP, and HR with ICP. Studies are needed to understand which variables from obesity have more influence over ICP and if HR can change ICP waveform morphology.

\section{Methods}

\section{Animals}

Initially, eighteen male Wistar rats 45-day-old healthy started 60 days of adaptation and were used to induce obesity after 105 days. However, two animals were ill at the start of treatment and were withdrawn from the study. The animals were kept in controlled environmental conditions with constant temperature $\left(24^{\circ} \pm 1^{\circ} \mathrm{C}\right)$, lighting ( $6 \mathrm{~h}$ light cycle / $18 \mathrm{~h}$ dark), and relative humidity $(55 \pm 5 \%)$. The animals had access to water and were fed through ad libitum. All procedures were approved by the Federal University to São Carlos (UFSCar) Animal Experimentation Ethics Committee (AEEC), CAEE - 3161311018)) and performed according to the regulations described in the Committee's Guiding Principles Manual. The entire experiment was carried out according to the ARRIVE 2.0 Guidelines.

\section{Experimental Design}

As previously described by Oishi et al. (2018) ${ }^{26}$ obesity was induced by the continuous supply of a highfat diet (18.12g of protein, $20 \mathrm{~g}$ of fat, $32.9 \mathrm{~g}$ of carbohydrates, and $2.97 \mathrm{~g}$ of fibers with $4.665 \mathrm{cal} / \mathrm{g} /$ $100 \mathrm{~g})$ for 24 weeks. In contrast, the non-obese animals were fed a standard diet $(22.81 \mathrm{~g}$ of protein, $4.80 \mathrm{~g}$ of fat, $39.0 \mathrm{~g}$ of carbohydrates, and 5.82 of fibers with $3.854 \mathrm{cal} / \mathrm{g} / 100 \mathrm{~g}$ ) during the same 24-week period. In this manner, the animals were randomized into the following groups Obese $(O B=9)$ and NonObese (NOB = 7), as shown in Fig. 1. The animals could not have any pathology, especially those that interfered with their eating behavior, cardiovascular system, or with great autonomic stress that would be removed from the study.

The characterization of the initial and final sample, as well as the differences between groups during the experiment, can be seen in Table 1 below. 
Table 1

Characterization of the samples, during the initial (W0) and final (W24) periods. $\mathrm{OB}=$ Obese, and NOB = Non Obese. Intracranial pressure ratio (ICP); Body mass (BM); Body fat (BF); Fat-free mass (FFM); Systolic blood pressure (SBP); Blood pressure (BP); diastolic blood pressure (DBP); mean arterial pressure (MAP); heart rate (HR). Paired t-

Student test for initial and final intra-group, and not paired in the same period, between groups. ${ }^{*} p<0.0001$, different from the initial intra-group period. ${ }^{*} p<0.0001$, differ rent from the final NOB group; $\$ p<0.05$ different from initial NOB group. ${ }^{1} B l o o d$ pressure and heart rate have their initial in week 12.

\begin{tabular}{|lllll|}
\hline \multicolumn{4}{|c}{ NOB } & \multicolumn{3}{l|}{ OB } \\
\hline & Initial & W24 & Initial & W24 \\
& Mean \pm sd & Mean \pm sd & Mean \pm sd & Mean \pm sd \\
\hline ICP (AU) & $0.65 \pm 0.10$ & $0.57 \pm 0.07$ & $0.66 \pm 0.12$ & $0.65 \pm 0.09^{\#}$ \\
\hline BM (g) & $440.9 \pm 9.4$ & $556.6 \pm 23.1^{*}$ & $442.9 \pm 11.0$ & $689.0 \pm 73.8^{* \#}$ \\
\hline BF (\%fat) & $13.21 \pm 2.45$ & $13.99 \pm 2.15$ & $10.29 \pm 2.21^{\$}$ & $24.79 \pm 4.45$ *\# \\
\hline FFM (g) & $382.6 \pm 14.1$ & $480.1 \pm 24.6 *$ & $396.9 \pm 13.4^{\$}$ & $519.1 \pm 34.9$ *\# \\
\hline${ }^{1} \mathrm{SBP}(\mathrm{mmHg})$ & $152 \pm 13$ & $139 \pm 11$ & $159 \pm 14$ & $141 \pm 2$ \\
\hline${ }^{1} \mathrm{DBP}(\mathrm{mmHg})$ & $117 \pm 11$ & $103 \pm 7$ & $120 \pm 13$ & $105 \pm 5$ \\
\hline${ }^{1} \mathrm{MAP}(\mathrm{mmHg})$ & $128 \pm 25$ & $114 \pm 8$ & $133 \pm 14$ & $120 \pm 8$ \\
\hline${ }^{1} \mathrm{HR}(\mathrm{bpm})$ & $315 \pm 26$ & $302 \pm 14$ & $353 \pm 22$ & $333 \pm 24^{\#}$ \\
\hline
\end{tabular}

\section{Data Analysis}

ICPn and dual-energy X-ray absorptiometry (DXA) were performed every 4 weeks on the same animal until the end of the experiment. However, tail plethysmography started at week 12, after obesity classified for $\mathrm{BM}$, subsequently following the same interval of 4 weeks. In this study, we used non-invasive measures to monitor and develop obesity and its comorbidities in the same animal, using DXA, which is considered the gold standard of assessment for body composition.

ICP analysis was performed using the Braincare 3.0 device (Braincare Corp). The equipment consists of capturing deformations and changes in the skull. The non-invasive sensor is attached to a bar in a stereotaxic and is associated with a tensiometer that captures the deformations of the skull and positions it over the parietal bone. At each evaluation, the animals were anesthetized with ketamine (80 $\mathrm{mg} / \mathrm{kg}$ ) and xylazine (32 $\mathrm{mg} / \mathrm{kg}$ ) to avoid movement. 
This evaluation model was previously evaluated and validated by Cabella et al. (2016) ${ }^{12}$ with a strong relation to the invasive method, demonstrating the waveform morphology of the ICP. The protocol experimental for assessment of waveform morphology of ICP can be seen in Fig. 2 below. Ten minutes of recordings were performed for each animal. The signals were recorded and later analyzed in the Braincare Analytics system, with the passage of filters for noise, breathing, and electrical signals. With passive analogical filter for anti-aliasing, and filtering network frequencies of $50 / 60 \mathrm{~Hz}$, to avoid interference from the power grid. During the analysis, the ICP data is subjected to a mathematical treatment comprising a $5 \mathrm{~Hz}$ low-pass filter. The capture of waveform morphology of ICP is carried out pulse by pulse, delivering a graph and averages for each minute of collection, shown in Fig. 7. However, for the software to average each minute, a minimum of 60 pulses are required for a minute. Therefore, the minutes with insufficient pulses or the minutes that the animal moved causing several artefacts were excluded. Next, the peak amplitude ratio (P2/P1) of each minute was established, and the average of this ratio was then calculated for the total analysis time of each animal. Thus, the representation of the peaks is as follows: P1 represents ventricular systole and P2 represents brain compliance ${ }^{6,20}$.

Body composition was determined by DXA analysis (Discovery A; Hologic Inc. - Beldford, MA, USA). The procedure consisted of evaluating the sedated animals, in the morning, and before the analysis of the ICP. The data obtained were subsequently analysed through the report, and we used the values of fat-free mass (FFM (g)), body mass (BM (g)), fat percentage (\%fat), and bone mineral content (BMC (g)) for analysis.

Systolic blood pressure (SBP), diastolic blood pressure (DBP), mean blood pressure (MAP) and heart rate (HR) were measured using a tail plethysmograph for rats and mice (Insight, V3.0) with a volume pressurerecording (VPR) sensor. A tube of measures similar to the restrictor was placed in the cages for adaptation of the animals 3 to 5 days before the evaluation. Data were collected with conscious animals, at rest and kept under movement restrictions, as previously described ${ }^{37}$. A total of 3 to 5 measurements were performed, with the average value being calculated.

\section{Statistical Analysis}

Statistical analysis was performed using the GraphPad Prism v.5 software. The Kolmogorov-Smirnov test was used to verify the homogeneity of the sample, using mean \pm standard deviation. The area under the curve was calculated and intergroup analysis of variance (Two-Way ANOVA) was performed with Bonferroni post-hoc (significance level adopted, $p<0.05$ ), for analyses performed during the 24 weeks. For DBP, SBP, MAP and HR an independent t-Student test was performed with a significance level of $p<$ 0.05 after calculating the area between weeks 12 and 24. Descriptive and frequency test was used to demonstrate the frequency of evaluated patterns, following physiological patterns predetermined by literature.

\section{Declarations}

\section{Statistical Analysis}


Statistical analysis was performed using the GraphPad Prism v.5 software. The Kolmogorov-Smirnov test was used to verify the homogeneity of the sample, using mean \pm standard deviation. The area under the curve was calculated and intergroup analysis of variance (Two-Way ANOVA) was performed with Bonferroni post-hoc (significance level adopted, $p<0.05$ ), for analyses performed during the 24 weeks. For DBP, SBP, MAP and HR an independent t-Student test was performed with a significance level of $p$ $<0.05$ after calculating the area between weeks 12 and 24. Descriptive and frequency test was used to demonstrate the frequency of evaluated patterns, following physiological patterns predetermined by literature.

\section{Acknowledgements}

This work was supported by a grant from the Brazilian agency National Council for Scientific and Technological Development (CNPQ) and by grants from the São Paulo Research Foundation (FAPESP grant 2016/09602-4).

\section{Author contributions statement}

D.A.M. conceived, and conducted the study, and analysed the results, J.M.A., M.S.F., C.A., S.M.M., J.A.T., V.O.F. and C.N.S.R. conducted the experiment(s), R.C.B., and A.C.G.O.D. analysed the results and conceived the experiment(s). All authors reviewed the manuscript.

\section{Additional information}

The authors declare no conflict of interest.

\section{References}

1. do Carmo, J. M. et al. Obesity-Induced Hypertension: Brain Signaling Pathways. Curr. Hypertens. Rep. 18, 1-9 (2016).

2. Szewka, A. J., Bruce, B. B., Newman, N. J. \& Biousse, V. Idiopathic intracranial hypertension: relation between obesity and visual outcomes. J. Neuroophthalmol. 33, 4-8 (2013).

3. Marmarou, A., Shulman, K. \& LaMorgese, J. Compartmental analysis of compliance and outflow resistance of the cerebrospinal fluid system. J. Neurosurg. 43, 523-34 (1975).

4. Xu, P., Kasprowicz, M., Bergsneider, M. \& Hu, X. Improved Noninvasive Intracranial Pressure Assessment With Nonlinear Kernel Regression. 14, 971-978 (2010).

5. Dunn, L. T. Raised intracranial pressure. J Neurol Neurosurg Psychiatry 73, 23-27 (2002).

6. Unnerbäck, Mårten, Ottensen Jhonny T., R. P. ICP curve morphology and intracranial flow-volume changes: a simultaneous ICP and cine phase-contrast MRI study in humans. Acta Neurochir. (Wien). 160, 219-224 (2018).

7. De-Lima-Oliveira, M. et al. intracranial Hypertension and Cerebral Autoregulation: A Systematic Review and. World Neurosurg. 113, 110-124 (2018). 
8. Sinclair, A. J. et al. Exploring the pathogenesis of IIH: An inflammatory perspective. J. Neuroimunology 201, 212-220 (2008).

9. Sinclair, A. J., et al. Low energy diet and intracranial pressure in women with idiopathic intracranial hypertension: prospective cohort study. BMJ, 341, c2701 (2010). doi: 10.1136/bmj.c2701. PMID: 20610512; PMCID: PMC2898925.

10. Andrews, L. E., Liu, G. T. \& Ko, M. W. Idiopathic intracranial hypertension and obesity. Horm. Res. Paediatr. 81, 217-225 (2014).

11. Cardim, D. A. et al. Characterization of ICP Behavior in an Experimental Model of Hemorrhagic Stroke in Rats. Acta Neurochir. Suppl. 122, 121-124 (2016).

12. Cabella, B. et al. Validation of a New Noninvasive Intracranial Pressure Monitoring Method by Direct Comparison with an Invasive Technique. Acta Neurochir. Suppl. 122, 93-96 (2016).

13. Rangel-Castillo, L. et al. Management of Intracranial Hypertension. Neurol Clin 26, 1-18 (2009).

14. Mascarenhas, S. et al. The New ICP Mnimally Invasive Method Shows that the Monro-Kellie doctrine is not valid. Acta Neurochir. Suppl. 114, 117-119 (2012).

15. Cardim, D. et al. Assessment of non-invasive ICP during CSF infusion test: an approach with transcranial Doppler. Acta Neurochir. (Wien). 158, 279-287 (2016).

16. Alperin, N. et al. MRI evidence of impaired CSF homeostasis in obesity-associated idiopathic intracranial hypertension. Am. J. Neuroradiol. 34, 29-34 (2013).

17. Frigieri, G. et al. Analysis of a Non-invasive Intracranial Pressure Monitoring Method in Patients with Traumatic Brain Injury. 126, 107-110 (2018).

18. Martin, N. A. \& Hu, X. Intracranial Pressure Signal Morphology. 49-52 (2012).

19. Kosmorsky, G. S. Idiopathic intracranial hypertension: Pseudotumor cerebri. Headache 54, 389-394 (2014).

20. Nucci, C. G. et al. Intracranial pressure wave morphological classification: automated analysis and clinical validation. Acta Neurochir. (Wien). 158, 581-588 (2016).

21. Czoniska, M. et al. Intracranial Pressure: More Than a number. Neurosurg Focus 22, 1-7 (2007).

22. Duarte, F. O. et al. Food restriction and refeeding induces changes in lipid pathways and fat deposition in the adipose and hepatic tissues in rats with diet-induced obesity. Exp. Physiol. 97, 88294 (2012).

23. Costa, R. R. S. et al. High fat diet induces central obesity, insulin resistance and microvascular dysfunction in hamsters. Microvasc. Res. 82, 416-422 (2011).

24. White, P. A. S. et al. Modelo de obesidade induzida por dieta hiperlipídica e associada à resistência à ação da insulina e intolerância à glicose. Arq. Bras. Endocrinol. Metabol. 57, 339-345 (2013).

25. Vileigas, D. F. et al. Saturated high-fat diet-induced obesity increases adenylate cyclase of myocardial $\beta$-adrenergic system and does not compromise cardiac function. Physiol. Rep. 4, e12914 (2016). 
26. Oishi, J. C. et al. Endothelial Dysfunction and Inflammation Precedes Elevations in Blood Pressure Induced by a High-Fat Diet. Arq. Bras. Cardiol. 558-567 (2018). doi:10.5935/abc.20180086

27. Gó Mez-Ambrosi, J. et al. Body mass index classification misses subjects with increased cardiometabolic risk factors related to elevated adiposity. Int. J. Obes. 36, 286-294 (2011).

28. Leopoldo, A. S., Nascimento, A. F., Luvizotto, R. A. M. \& Sugizaki, M. M. Classification of different degrees of adiposity in sedentary rats. 49, 1-9 (2016).

29. Shih, K. \& Kwok, C. Exercise reduces body fat and improves insulin sensitivity and pancreatic $\beta$-cell function in overweight and obese male Taiwanese adolescents. 1-8 (2018). doi:10.1186/s12887018-1025-y

30. Yanagihara, G. R. et al. Influence of high fat diet on bone tissue: an experimental study in growing rats. J Ntur Heal. Aging 3, 1-7 (2016).

31. Souza, R. G. M. de, Gomes, A. C., Prado, C. M. M. do \& Mota, J. F. Métodos de análise da composição corporal em adultos obesos Methods for body composition. Rev. Nutr. 27, 569-583 (2014).

32. Uldall, M., Bhatt, D. K., Kruuse, C., Juhler, M. \& Jensen, R. H. Choroid plexus aquaporin 1 and intracranial pressure are increased in obese rats: towards an idiopathic intracranial hypertension model? 41, 1141-1147 (2017).

33. Markey, K. A., Mollan, S. P., Jensen, R. H. \& Sinclair, A. J. Understanding idiopathic intracranial hypertension: mechanisms, management, and future directions PROOF. 78-91 (2016). doi:10.1016/S1474-4422(15)00298-7

34. Shiou, Y., Huang, I. \& Lin, H. High fat diet aggravates atrial and ventricular remodeling of hypertensive heart disease in aging rats. J. Formos. Med. Assoc. 117, 621-631 (2018).

35. Feng, M. et al. Validation of volume-pressure recording tail-cuff blood pressure measurements. Am J of Hypertension 21, 1288-1291 (2008).

36. Speretta, G. F. et al. High-fat diet increases respiratory frequency and abdominal expiratory motor activity during hypercapnia. Respir Physiol Neurobio/ 258, 32-39 (2018).

37. Maglione, A.V., et al. Impact of swimming exercise onf inflamation in medullary areas of sympathetic outflow control in spontaneously hipertensive rats. Metabolic Brain Disease 33, 1649-1660 (2018).

\section{Figures}

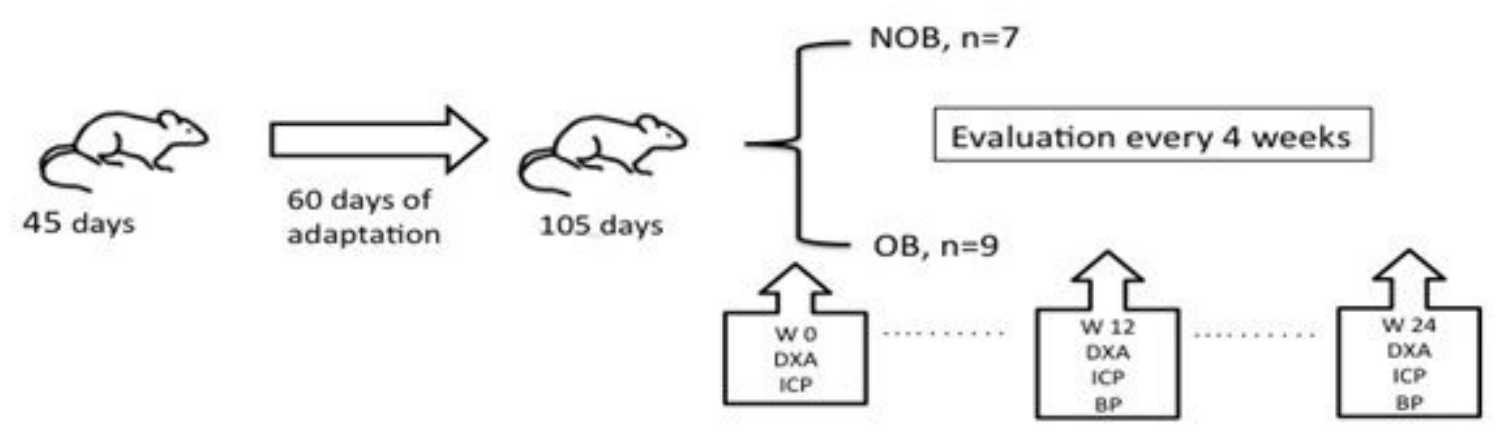


Figure 1

Experimental design. Arrival, adaptation to the environment and induction of obesity after adulthood. Assessments every 4 weeks, however, with tail plethysmography for blood pressure (BP) starting at week 12.

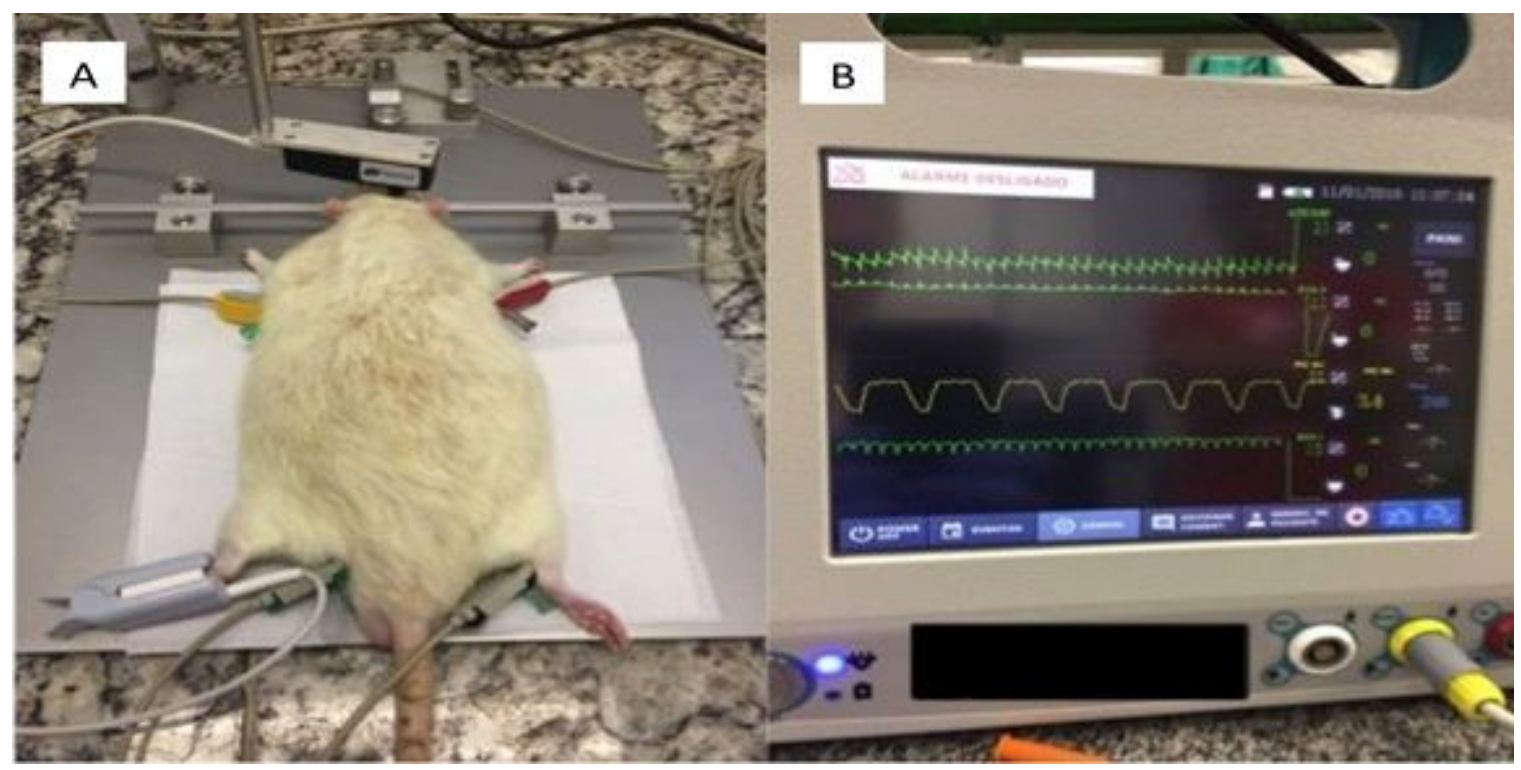

Figure 2

Experimental protocol. 2A: sedated animal attached to the stereotaxic, with a sensor over the parietal lobe. 2B: pulse-to-pulse data recording. ICP waveform morphology sign (yellow).
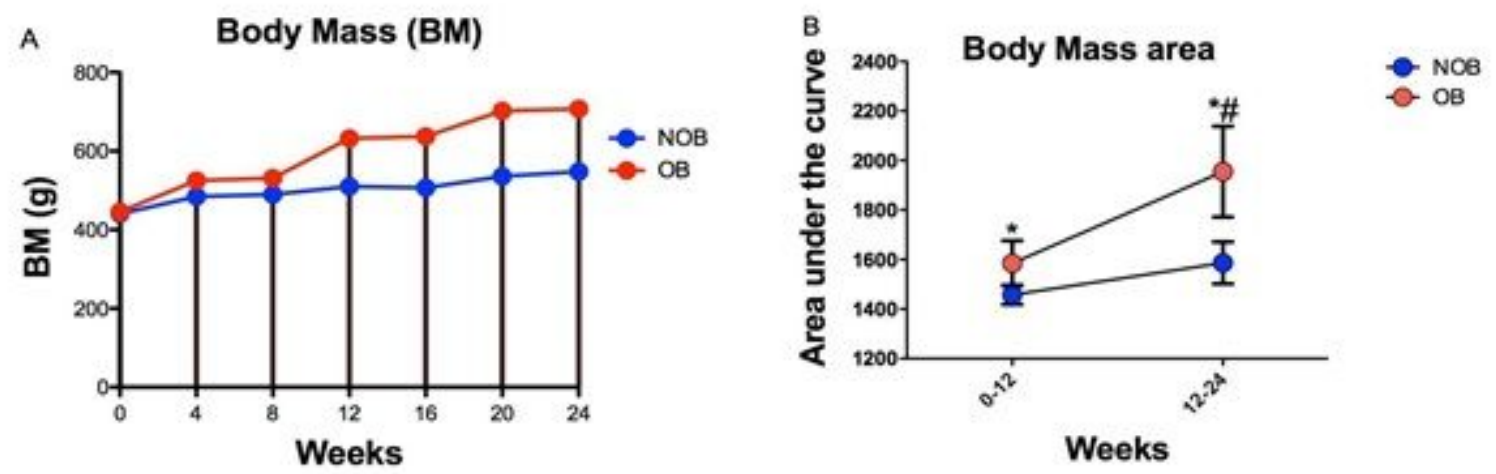

\section{Figure 3}

$\mathrm{NOB}=$ Non Obese rats (blue); $\mathrm{OB}=$ Obese rats (red). $3 \mathrm{~A}$ Body mass development over 24 weeks (group averages). 3B Area under the curve between periods 0 - 12 weeks and between 12 - 24 weeks with twoway ANOVA with Bonferroni post-hoc. *Different between weeks of the same period $(p<0.05$, period at 0 12 weeks and $p<0.001$ at 12 - 24 weeks) \#different between groups $(p<0.001)$. 

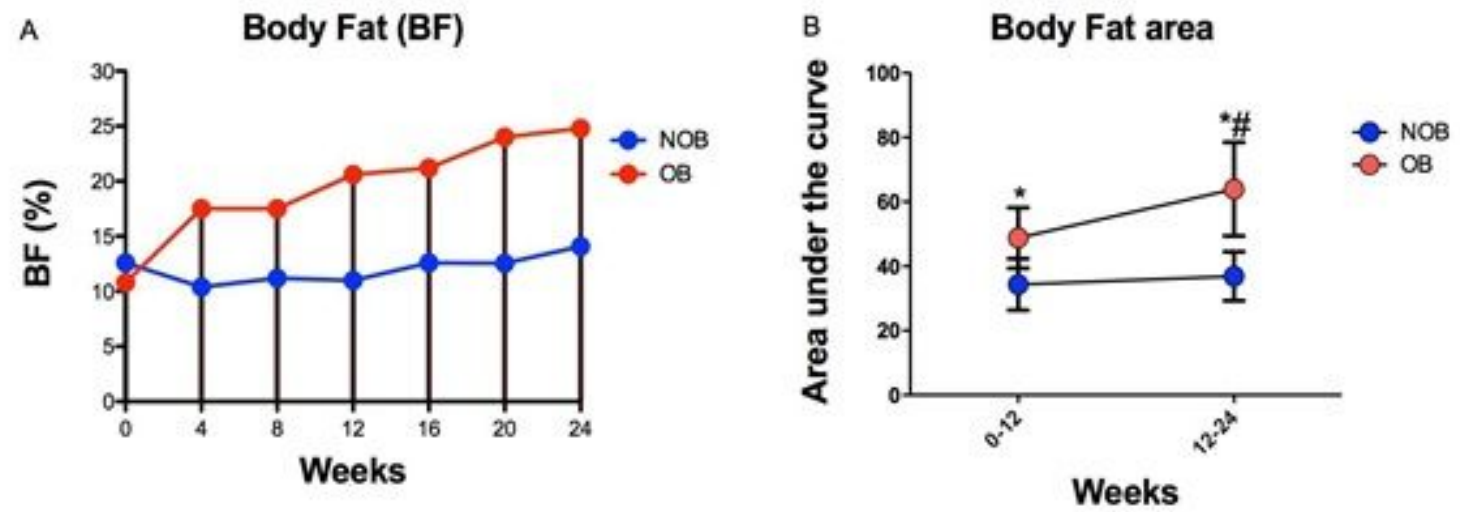

\section{Figure 4}

$\mathrm{NOB}=$ Non Obese rats (blue); $\mathrm{OB}=$ Obese rats (red). $4 \mathrm{~A}$ Development of fat percentage over 24 weeks (group averages). 4B Area under the curve between periods 0 - 12 weeks and between 12 - 24 weeks. Twoway ANOVA with Bonferroni's post-hoc. *Different between weeks of same period $(p<0.05$, period at $0-12$ weeks and $p<0.001$ at 12 - 24 weeks) \#different between groups $(p<0.001)$.
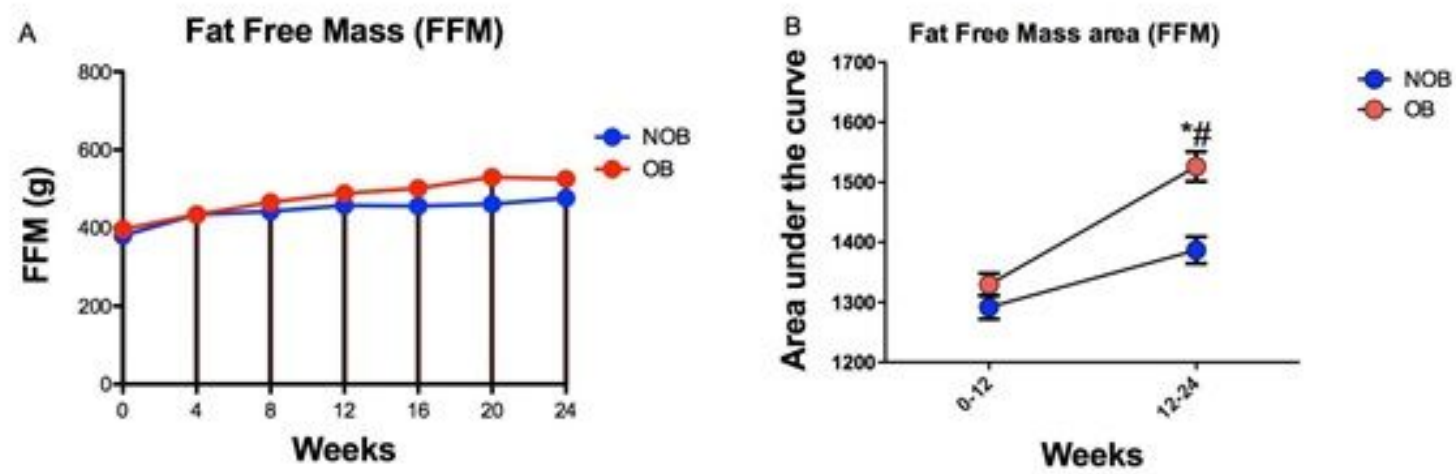

Figure 5

$\mathrm{NOB}=$ Non Obese rats (blue); $\mathrm{OB}=$ Obese rats (red). 5A FFM development over 24 weeks. 5B Area under the curve between 0 - 12 weeks and between 12 - 24 weeks. Two-way ANOVA with Bonferroni's post-hoc. *Different between weeks of same periods ( $p<0.001$, period at $12-24$ weeks) \#different between groups $(p<0.001)$. 

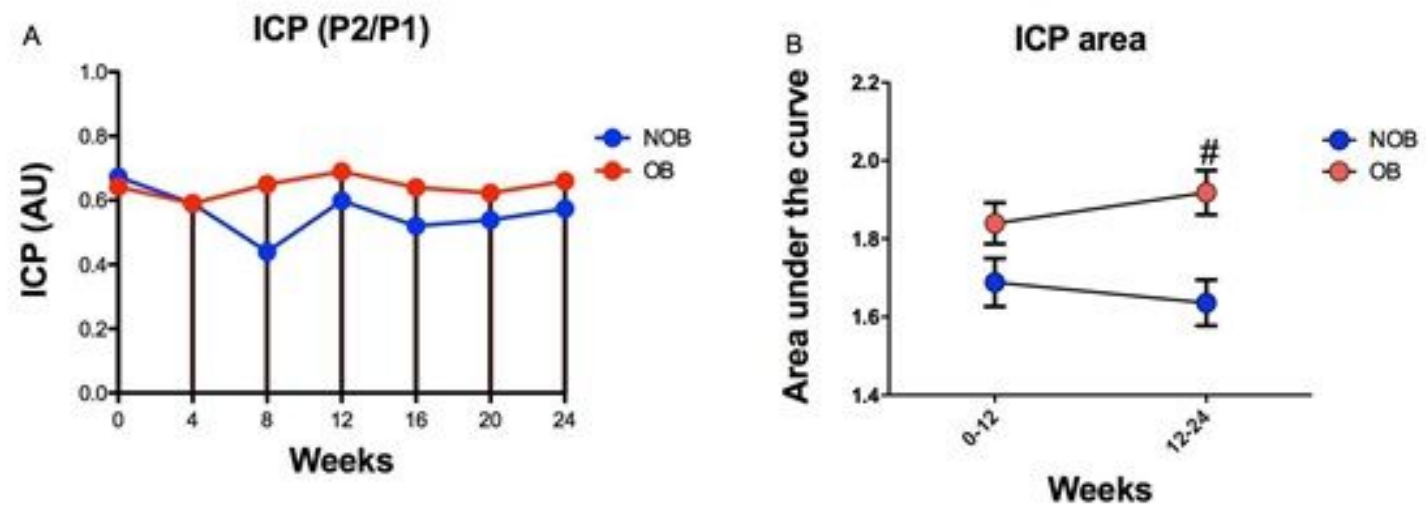

Figure 6

$\mathrm{NOB}=$ Non Obese rats (blue); $\mathrm{OB}=$ Obese rats (red). 6A Development of ICP ratio over 24 weeks. 6B Sum of areas between periods 0 - 12 weeks and between 12 - 24 weeks. Two-way ANOVA with Bonferroni's post-hoc. \#Different between groups $(p<0.001)$.
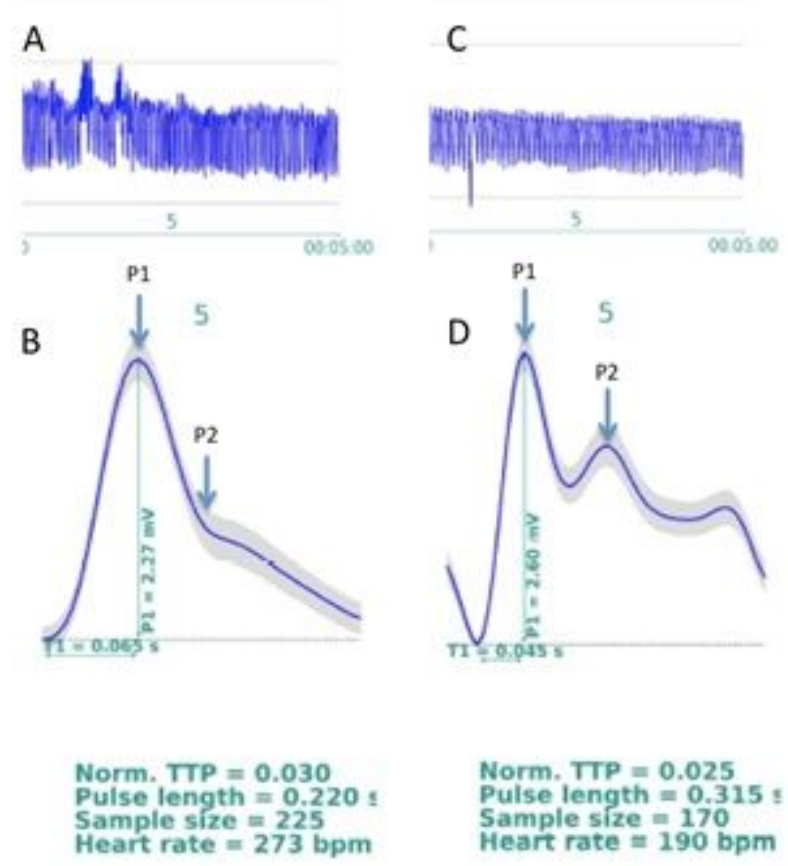

\section{Figure 7}

ICP waveform morphology analysed by system at 24 week. A, B) Continuous sampling of the ICP waveform morphology of non-obese rats (NOB) of the fifth minute. $C$; $D$ ) Continuous sampling of the ICP waveform morphology of obese rats (OB) of the fifth minute. $A ; C)$ Signals collected pulse by pulse for 1 minute; B; D) Average pulse per minute showing the collected values, such as P1 amplitude, P1 time (t1), as well as sample size, pulse length, heart rate, and time to peak (TTP). 

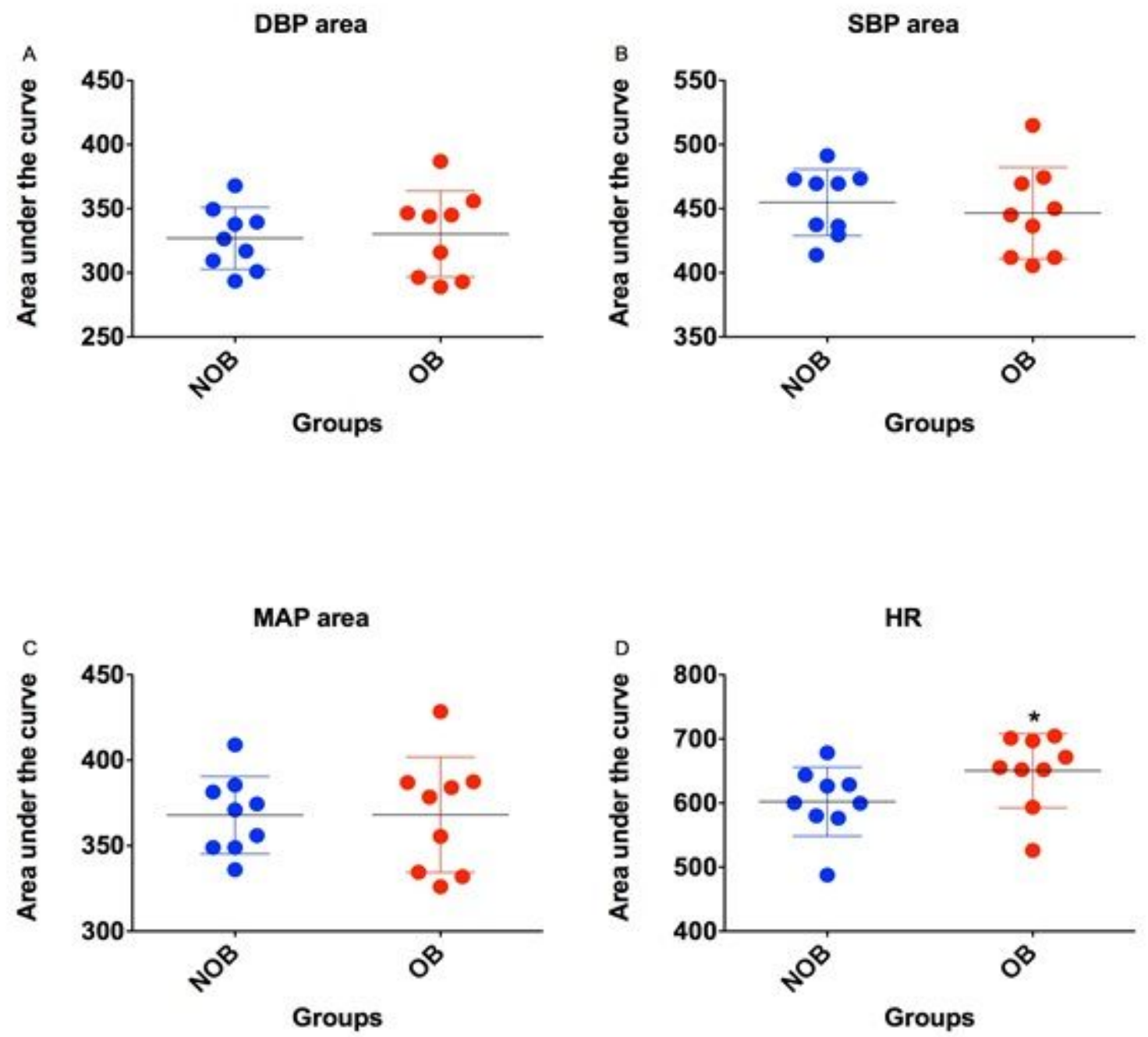

Figure 8

$\mathrm{NOB}=$ Non Obese rats (blue); $\mathrm{OB}=$ Obese rats (red). Area under the curve between weeks 12 and 24. 8A Diastolic Blood Pressure (DBP), 8B Systolic Blood Pressure (SBP), 8C Mean Arterial Pressure (MAP), 8D Heart Rate (HR). *Difference between groups $(p<0,05)$.

\section{Supplementary Files}

This is a list of supplementary files associated with this preprint. Click to download.

- Suplementaryinformation.docx 